\title{
Primary hepatic schwannoma: imaging and histological findings
}

\author{
HL Tsui *, SM Yu, CH Lau, Sherman SM Lam, PY Chu, YH Hui, KL Lo
}

Hong Kong Med J 2020;26:449.e1-4

https://doi.org/10.12809/hkmj198190

Schwannoma is a rare tumour in the liver. It is likely to arise from the hepatobiliary nerves among the hepatic plexus in the liver hilum as well as interlobular connective tissues and hepatic arteries. To the best of our knowledge, no prior publications have reported cases in Hong Kong.

We report the case of a 64-year-old man with a history of nasopharyngeal carcinoma and colon carcinoma and a new liver lesion detected on follow-up imaging for surveillance in 2018 following detection of a slightly elevated serum carcinoembryonic antigen level $(4.7 \mu \mathrm{g} / \mathrm{L})$. Alpha fetoprotein level was within the normal range (3.9 $\mu \mathrm{g} / \mathrm{L})$. Liver function tests were normal and he was asymptomatic with no history of neurofibromatosis.

Triphasic contrast computed tomography (CT) of the liver revealed a 5.2-cm ovoid hypodense lesion with heterogeneous enhancement in the caudate lobe of the liver (Fig 1). No washout of contrast was evident in the portal venous or delayed phases. Fluorodeoxyglucose-18 positron

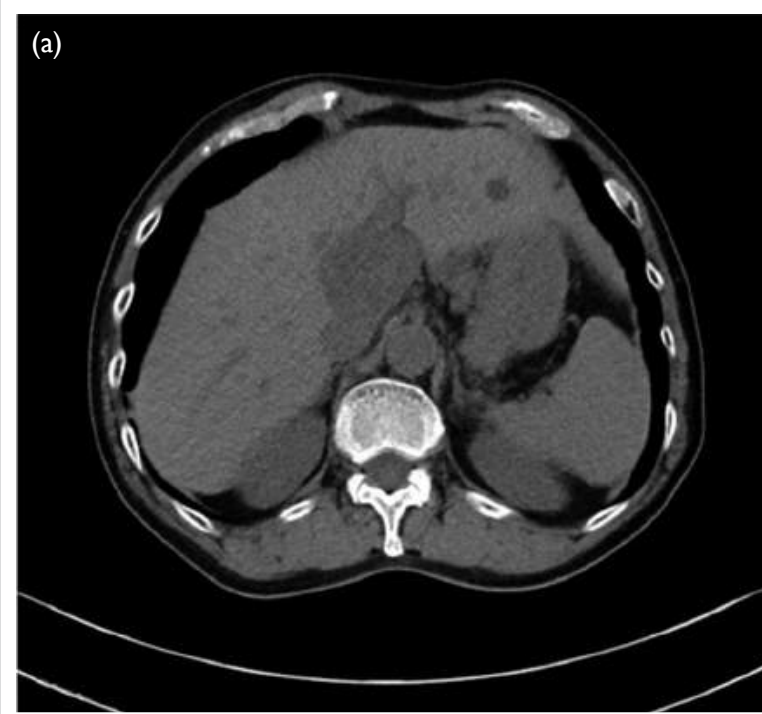

(b)
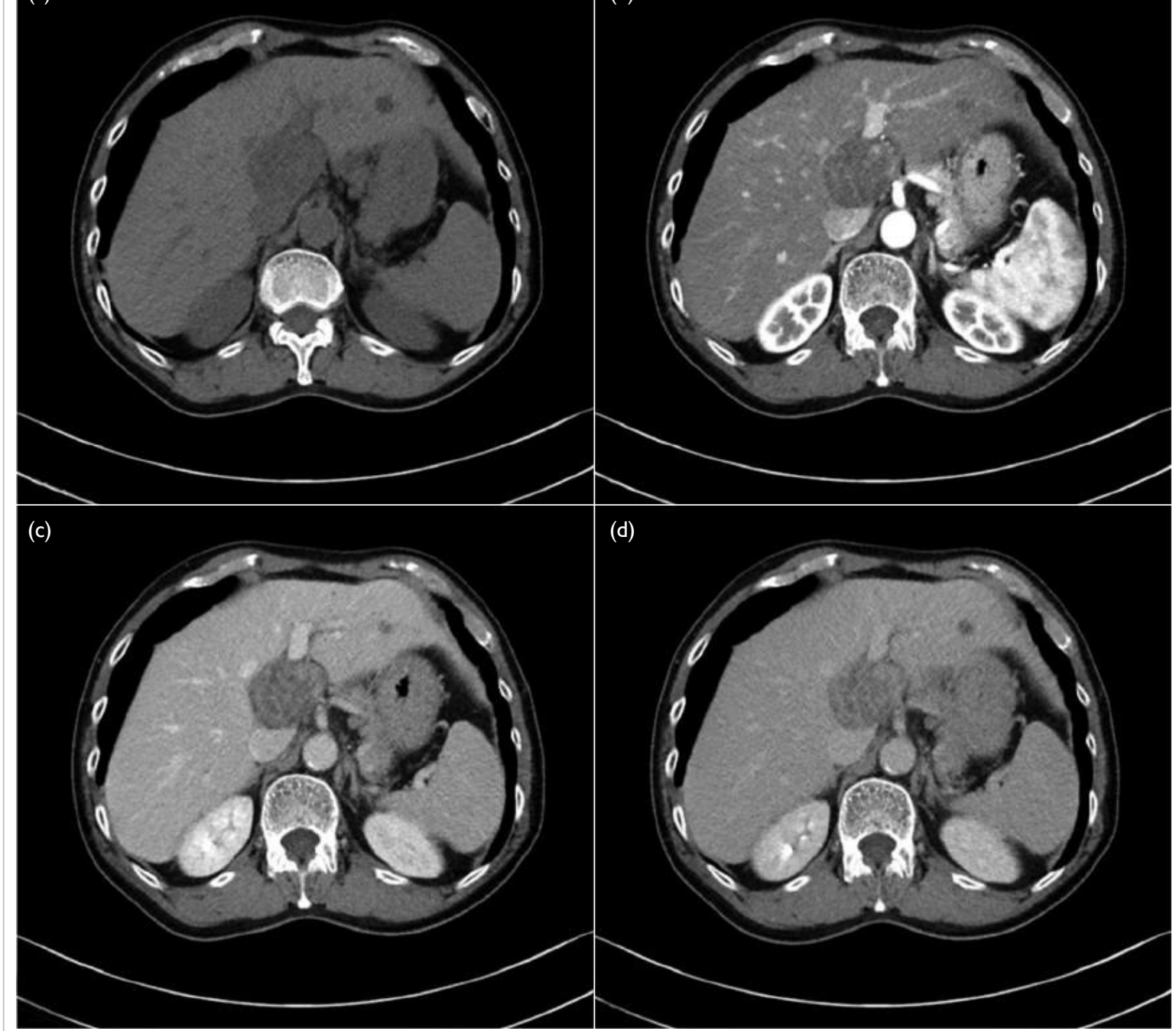

(d)

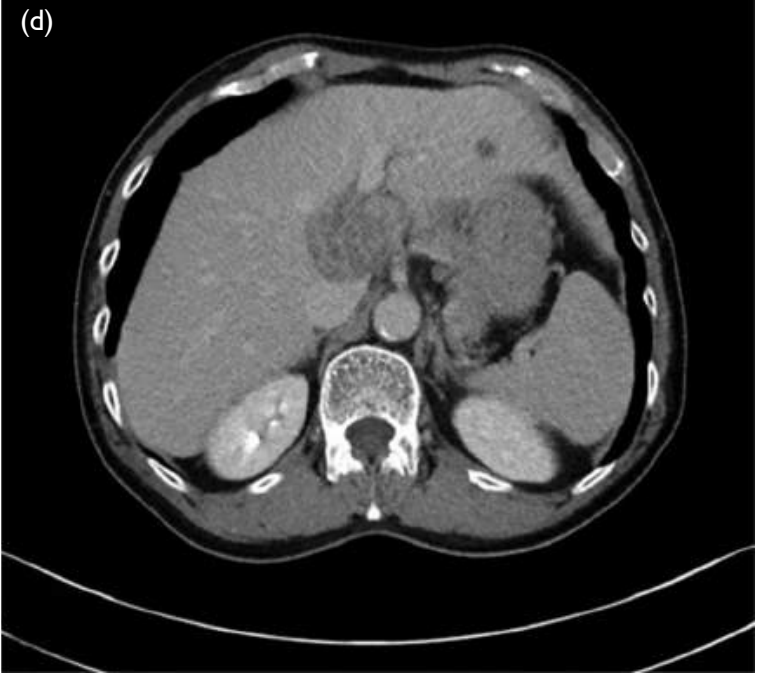

FIG I. Triphasic computed tomography scans in (a) pre-contrast, (b) arterial, (c) portal venous, and (d) delayed phases, showing a well-defined, ovoid hypodense lesion with heterogeneous enhancement that persists in portal venous and delayed phases in the caudate lobe of the liver 


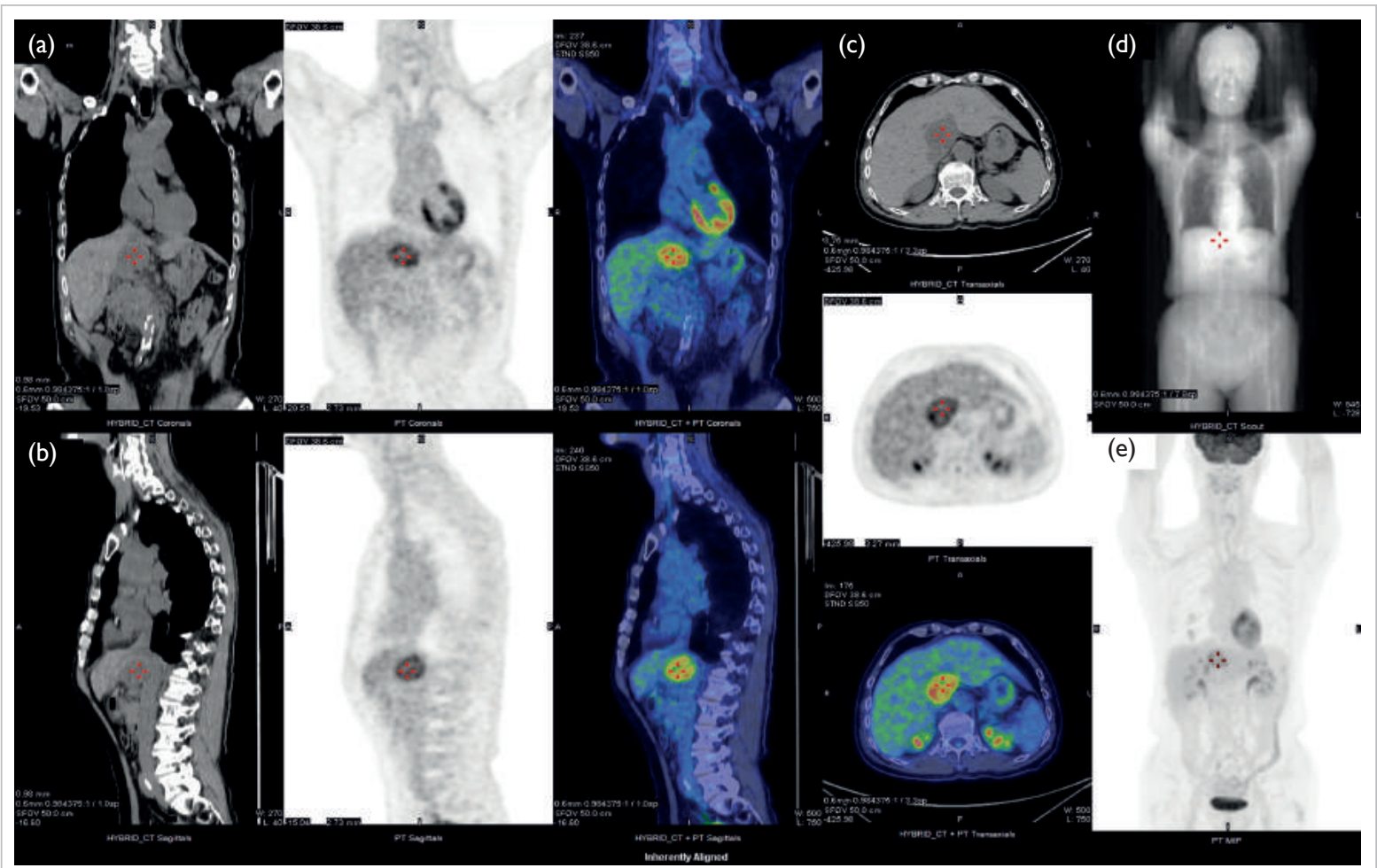

FIG 2. Positron emission tomography-computed tomography scan in (a) coronal, (b) sagittal and (c) axial planes together with the (d) scout and (e) maximum intensity projection images, showing a moderately hypermetabolic lesion at the caudate lobe of the liver (red crosses)

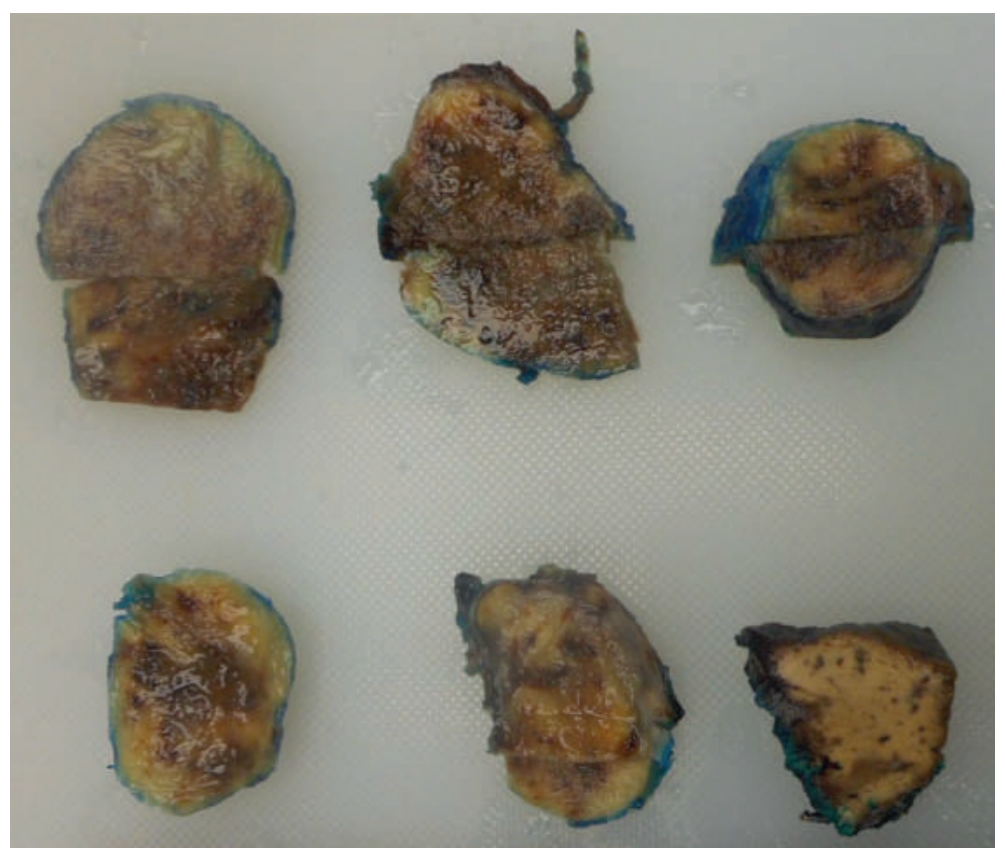

FIG 3. Gross specimen of hepatic resection with a well-circumscribed tumour mass and light tan colour cut surface
Surgical resection of the lesion was performed (Fig 3). Pathology showed a schwannoma and degenerative changes. Histological examination revealed an encapsulated tumour consisting of highly ordered Antoni type A and B areas (Fig 4). Immunohistochemical analysis showed the tumour cells to be diffusely positive for S100, consistent with neural differentiation (Fig 4). HerPar1, a mitochondrial antigen of hepatocytes, was negative. The CD34, a cell surface glycoprotein that is positive in gastrointestinal stromal tumour, was also negative.

Schwannoma is most commonly found in the limbs and the head and neck region. A fifth of cases shows association with neurofibromatosis type 1 . The mediastinum and retroperitoneum are other possible sites. It is uncommon in the gastrointestinal tract and extremely rare in the liver. ${ }^{1}$ It was first reported in 1978 by Pereira et al. ${ }^{2}$ A literature search through PubMed and MEDLINE revealed 32 reported cases. No cases have been published in Hong Kong.

The origin of hepatic schwannoma is the hepatobiliary nerves among the hepatic plexus in the liver hilum as well as interlobular connective tissues and the hepatic arteries. ${ }^{1,3}$ They are usually well-encapsulated and grow very slowly, usually smaller than $5 \mathrm{~cm}$ at the time of diagnosis. Larger schwannomas may undergo secondary degeneration with consequent pseudocystic regression, emission tomography-CT showed a moderately hypermetabolic lesion at the caudate lobe with a maximum standardised update value of 5.8 (Fig 2). 


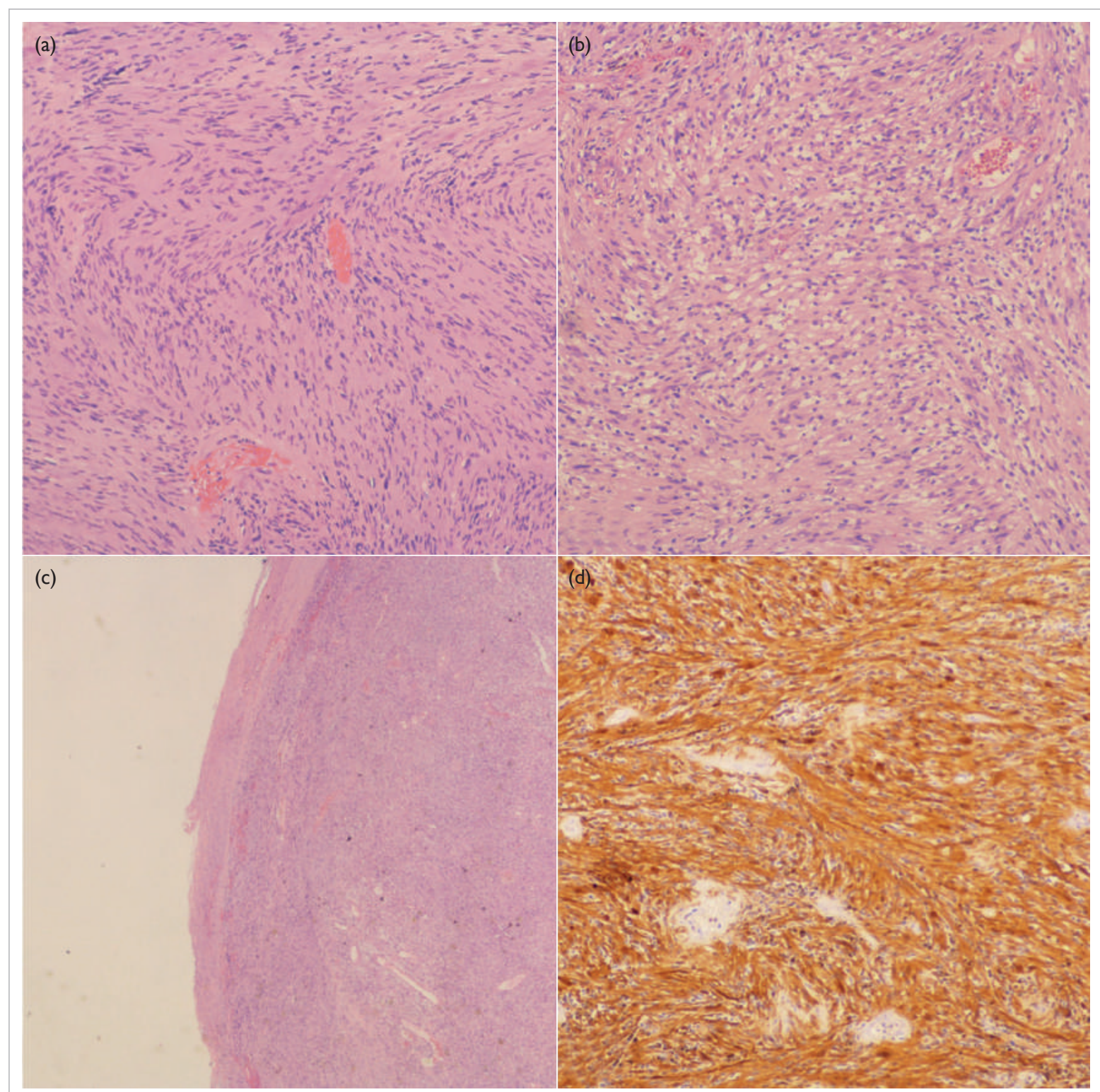

FIG 4. Micrographs of the tumour, showing (a) Antoni type A area, $\times 100$; (b) Antoni type $B$ area, $\times 100$; (c) circumscribed and encapsulated tumour, $\times 20$; and (d) immunostaining for S100 diffusely positive for the tumour cell, $\times 100$

haemorrhage, and calcification. Malignant CT, hypointense on T1-weighted, and hyperintense transformation is very rare. ${ }^{3}$

Pathologically, a schwannoma is an on T2-weighted magnetic resonance imaging. ${ }^{5}$ encapsulated tumour that arises within the nerve but there are no distinct radiological features that sheaths. It consists of a highly ordered cellular differentiate them from benign tumours. ${ }^{3}$ A hepatic component (Antoni type A area) characterised by schwannoma may be fluorodeoxyglucose-avid spindle cells with twisted nuclei arranged in short depending on inflammatory activity and cellularity. bundles, and a hypocellular area in a loose myxoid Fluorodeoxyglucose-18 positron emission stroma (Antoni type B area) that comprises a loose tomography-CT alone may enable differentiation meshwork of gelatinous and microcystic tissue. ${ }^{4}$

On imaging, hepatic schwannoma is liver. $^{1}$ usually well circumscribed with various signal Hepatic schwannoma is an extremely rare characteristics, depending on the distribution of tumour and preoperative diagnosis with imaging is Antoni A and Antoni B areas. ${ }^{1}$ It is commonly of challenging. Biopsy or surgical resection is usually low density with heterogeneous enhancement on required for definitive diagnosis. 


\section{Author contributions}

Concept or design: All authors.

Acquisition of data: HL Tsui and CH Lau.

Analysis or interpretation of data: HL Tsui, SM Yu, and CH Lau.

Drafting of the manuscript: HL Tsui, SM Yu, and CH Lau.

Critical revision of the manuscript for important intellectual content: All authors.

All authors had full access to the data, contributed to the study, approved the final version for publication, and take responsibility for its accuracy and integrity.

\section{Conflicts of interest}

All authors have disclosed no conflicts of interest.

\section{Funding/support}

This research received no specific grant from any funding agency in the public, commercial, or not-for-profit sectors.

\section{Ethics approval}

This study is approved by the cluster Research Ethics Committee (Ref KC/KE-19-0247/ER-3). Written patient consent was also obtained.

${ }^{1}$ HL Tsui *, MB, ChB, FRCR

${ }^{1} \mathrm{SM}$ Yu, MB, ChB, FHKAM (Radiology)
${ }^{2} \mathrm{CH}$ Lau, MB, ChB

${ }^{3}$ SSM Lam, MB, BS, FHKAM (Surgery)

${ }^{1}$ PY Chu, MB, ChB, FHKAM (Radiology)

${ }^{1}$ YH Hui, MB, BS, FHKAM (Radiology)

${ }^{1}$ KL Lo, MB, ChB, FHKAM (Radiology)

1 Department of Radiology and Organ Imaging, United Christian Hospital, Hong Kong

${ }^{2}$ Department of Pathology, United Christian Hospital, Hong Kong

${ }^{3}$ Department of Surgery, United Christian Hospital, Hong Kong

* Corresponding author: karen.tsuihl@gmail.com

\section{References}

1. Hayashi M, Takeshita A, Yamamoto $\mathrm{K}$, Tanigawa N. Primary hepatic benign schwannoma. World J Gastrointest Surg 2012;4:73-8.

2. Pereira Filho RA, Souza SA, Oliveira Filho JA. Primary neurilemmal tumour of the liver: case report. Arq Gastroenterol 1978;15:136-8.

3. Ozkan EE, Guldur M, Uzunkoy A. A case report of benign schwannoma of the liver. Intern Med 2010;49:1533-6.

4. Wan DL, Zhai ZL, Ren KW, Yang YC, Lin SZ, Zheng SS. Hepatic schwannoma: a case report and an updated 40 -year review of the literature yielding 30 cases. Mol Clin Oncol 2016;4:959-64.

5. Yamamoto M, Hasegawa $K$, Arita J, et al. Primary hepatic schwannoma: a case report. Int J Surg Case Rep 2016;29:146-50. 